\title{
Rotura y desplazamiento de catéter arterial umbilical: obstrucción arterial bilateral en un prematuro de muy bajo peso al nacer
}

\author{
Rupture and displacement of umbilical catheter: bilateral arterial occlusion in \\ VLBW preterm
}

\author{
Dra. Dilek Dilli , Dra. Elif Özyazici ${ }^{a}$,Dra. Nurdan Fettah ${ }^{a}$, Dr. Özkan Kayab, Dra. Melek Pala Akdoğanc, \\ Dra. Ayşegül Zenciro lua, Dr. Nurullah Okumuşs y Dra. Nilüfer Güzoğlud
}

\begin{abstract}
RESUMEN
La canalización de los vasos umbilicales es un procedimiento frecuente en las unidades de cuidados intensivos neonatales, especialmente en los recién nacidos de muy bajo peso al nacer. Raras veces el catéter arterial umbilical se rompe; los fragmentos retenidos pueden provocar trombosis, infección, embolización distal e incluso la muerte. En este artículo, describimos el caso de un recién nacido con isquemia bilateral, clínicamente significativa, de las extremidades que se manifestó después de la extracción de un catéter arterial umbilical roto. Estaba recibiendo tratamiento vasodilatador junto con fibrinolíticos y anticoagulantes. La evolución fue favorable.

Palabras clave: lactantes prematuros, catéter, trombosis.
\end{abstract}

http:/ /dx.doi.org/10.5546/aap.2015.e283

\section{INTRODUCCIÓN}

La canalización de los vasos umbilicales es un procedimiento frecuente en las unidades de cuidados intensivos neonatales (UCIN), especialmente en los recién nacidos de muy bajo peso al nacer. El catéter arterial umbilical (CAU) está indicado para el análisis de gases en sangre o de muestras en el laboratorio y para el monitoreo continuo de la presión arterial. ${ }^{1,2}$ Raras veces, el CAU se rompe y los fragmentos retenidos pueden

a Departamento de Neonatología

b. Departamento de Cardiología Pediátrica.

c. Departamento de Radiología.

Hospital Universitario y de Investigación Materno Infantil Dr. Sami Ulus, Angora, Turquía.

d. Departamento de Neonatología, Facultad de Medicina, Universidad Kırıkkale.

Correspondencia:

Dra. Dilek Dilli: dilekdilli2@yahoo.com

Financiamiento: Ninguno.

Conflicto de intereses: Ninguno que declarar.

Recibido: 2-2-2015

Aceptado: 5-4-2015 provocar trombosis, infección, embolización distal e incluso la muerte. ${ }^{3-6}$ Además, es frecuente el diagnóstico de trombosis neonatal asociada con el uso de sondas intravasculares permanentes. ${ }^{7-9}$

Describimos el caso de un recién nacido con isquemia bilateral clínicamente significativa de las extremidades que se manifestó después de la extracción de un CAU roto. En ese momento recibía tratamiento vasodilatador junto con fibrinolíticos y anticoagulantes. La evolución fue favorable.

\section{CASO CLÍNICO}

Se trasladó a un recién nacido de sexo masculino nacido a las 28 semanas de gestación con un peso de $1000 \mathrm{~g}$ desde otro hospital a nuestra UCIN debido a la rotura de un catéter arterial umbilical al tercer día de vida. Había sido ingresado por prematurez y dificultad respiratoria a una UCIN regional, donde se le colocaron catéteres en arteria y vena umbilicales. A los tres días de vida, se le retiró el catéter venoso umbilical debido a fuga de líquido. Se cortó el CAU con un bisturí a nivel de la piel mientras se retiraba la sutura de sujeción. Se realizaron varios intentos para recuperar el fragmento roto con una pinza quirúrgica, pero no logró visualizarse. Inmediatamente, derivaron al paciente a nuestra unidad. Una radiografía simple mostró un fragmento de $11 \mathrm{~cm}$ del CAU roto desde el origen de la arteria umbilical hacia el cayado aórtico distal. Se hizo una consulta con cardiólogos pediátricos para determinar el tratamiento ideal. Se trasladó al paciente a la sala de angiografía, donde se realizó un abordaje transfemoral sin éxito. Luego, se insertó un catéter de cuello de cisne con lazo, bajo guía fluoroscópica, en la arteria umbilical derecha. Tras sujetar el extremo del CAU roto, se lo retiró satisfactoriamente. Transcurrieron seis horas desde la rotura del catéter hasta el inicio del procedimiento intravascular. 
El paciente fue trasladado nuevamente a la UCIN, donde permaneció estable durante el primer día posterior al procedimiento de extracción. Al día siguiente, presentó isquemia bilateral de las extremidades inferiores; no se le detectaron los pulsos de las arterias femorales y distales (Figura 1). Mediante una ecografía doppler se observó la obstrucción bilateral de la arteria ilíaca externa que comprometía las arterias femoral, poplítea y crural. Debido a la progresión rápida de la isquemia en las extremidades, se decidió instaurar un protocolo terapéutico con un activador recombinante del plasminógeno tisular (APTr) (una inyección intravenosa rápida inicial de $0,7 \mathrm{mg} / \mathrm{kg}$ durante 30 a 60 minutos seguida por una infusión de $0,2 \mathrm{mg} / \mathrm{kg} / \mathrm{h}$ ) y un goteo de heparina a dosis baja (10 UI/ $\mathrm{kg} / \mathrm{h})$, además de infusiones con plasma congelado en fresco. ${ }^{8} \mathrm{La}$ concentración sérica de fibrinógeno y el tiempo de tromboplastina parcial activado (TTPa) fueron normales. No se observó un cambio en el color de la extremidad, por lo que se inició una infusión intravenosa con nitroglicerina $(0,5 \mu / \mathrm{kg} / \mathrm{min})$ y se aplicó una pomada tópica de nitroglicerina al $2 \%$ ( $4 \mathrm{~mm} / \mathrm{kg}$ tres veces al día) en todas las superficies isquémicas. En los días siguientes se observó una mejoría gradual en el color y el llenado capilar. La superficie isquémica se limitó a las puntas de los dedos de los pies.

A las 72 horas de tratamiento, una ecografía doppler mostró la presencia de circulación colateral y de flujo sanguíneo pulsátil en los grandes vasos. El mismo día, el paciente presentó movimientos convulsivos. La ecografía craneal previa al tratamiento fibrinolítico fue normal.

FIGURA 1. Isquemia bilateral de las extremidades inferiores tras la extracción del fragmento roto del catéter

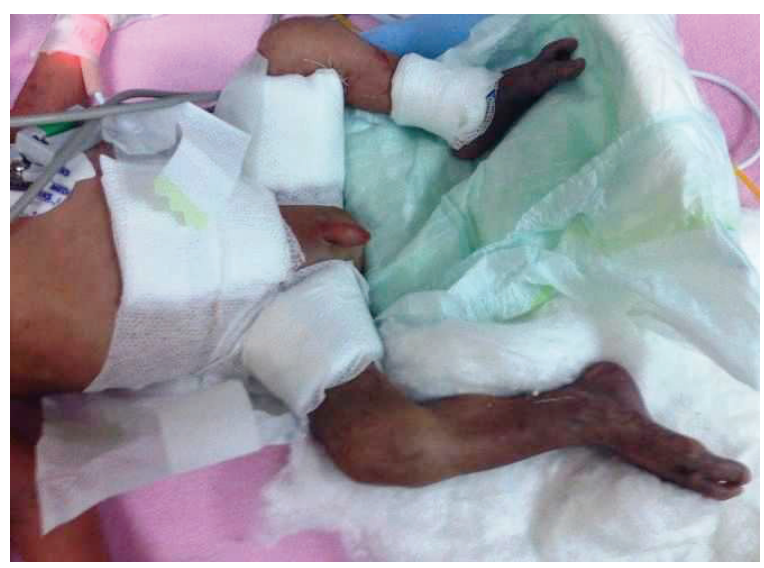

No obstante, la ecografía craneal de control mostró hemorragia intracraneal de grado 3. En consecuencia, se interrumpió la infusión intravenosa de nitroglicerina y el APTr, y se disminuyó la dosis de heparina a $5 \mathrm{UI} / \mathrm{kg} / \mathrm{h}$. Al séptimo día de tratamiento, el goteo de heparina se reemplazó por otro de heparina de bajo peso molecular (HBPM) hasta lograr un ajuste de la dosis a 0,5-1 UI/ml del anti-factor Xa.

Se interrumpió la aplicación tópica de nitroglicerina tras la recuperación total de los dedos de los pies, a los 15 días de iniciado el tratamiento (Figura 2). La ecografía craneal realizada a las ocho semanas de vida mostró hemorragia de grado 2 y leucomalacia periventricular.

El paciente no tuvo ninguna otra complicación y fue dado de alta aproximadamente a las 10 semanas de vida, conforme a los protocolos de nuestra UCIN. Continuó con HBPM durante seis semanas bajo estricta supervisión. A los tres meses de vida, su desarrollo neurológico y motriz era adecuado para su edad.

\section{DISCUSIÓN}

La rotura y embolización de los catéteres umbilicales son poco frecuentes, pero pueden tornarse en una situación de mal pronóstico. En la bibliografía se informaron casos de recuperación transvascular o quirúrgica de un CAU roto. ${ }^{4-6}$ Choi y cols., ${ }^{4}$ fueron los primeros en informar la rotura de un CAU en dos casos en 1977. Lograron recuperar el fragmento roto mediante la disección de la arteria ilíaca primitiva derecha. Murphy y cols., ${ }^{6}$ emplearon el abordaje transumbilical. En nuestro caso, el fragmento roto se embolizó en la aorta y se recuperó satisfactoriamente mediante el abordaje transumbilical.

FIgURA 2. Resolución completa 15 dias después del incidente

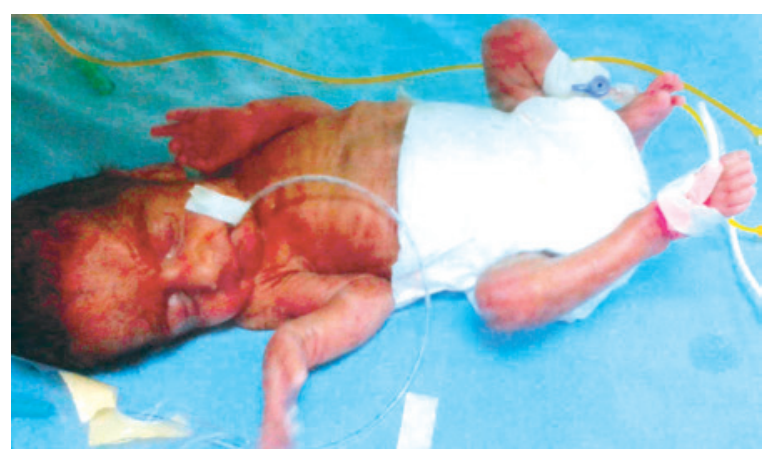


Por otro lado, en los recién nacidos prematuros, el diámetro arterial es pequeño en relación con el tamaño del catéter; por lo tanto, son más susceptibles a sufrir complicaciones, como vasoespasmo y trombosis con posterior isquemia tisular y necrosis de la extremidad afectada. En este caso, la trombosis pudo haber estado relacionada con el acceso umbilical, la intervención de la arteria femoral o el diámetro del equipo utilizado para el procedimiento de extracción del catéter.

El tratamiento habitual de las lesiones isquémicas implica la extracción inmediata del catéter, mantener la extremidad afectada elevada y aplicar compresas tibias en la extremidad contralateral (vasodilatación refleja). Principalmente, se utilizan anticoagulantes, como heparina no fraccionada o HBPM y trombólisis con APTr cuando se produce la obstrucción trombótica completa del vaso con isquemia de rápida progresión, y se considera que los riesgos asociados con una cirugía son considerables o incluso perjudiciales. ${ }^{7}$

La nitroglicerina es un conocido relajante del músculo liso que se absorbe rápidamente a través de la piel intacta. ${ }^{10}$ Forma el radical libre óxido nítrico, que activa la guanilato-ciclasa e incrementa el 3'5'-monofosfato cíclico de guanosina celular. De esta manera, se produce la desfosforilación de la miosina del músculo liso, que regula el estado contráctil y causa la vasodilatación. La dosis inicial habitual de $4 \mathrm{~mm} / \mathrm{kg}$ de pomada de nitroglicerina al $2 \%$, es equivalente a $0,2-0,5 \mathrm{mg} / \mathrm{kg} / \mathrm{min}$ administrada por vía intravenosa. ${ }^{10}$. En el caso que presentamos, las dosis repetidas de nitroglicerina tópica aliviaron la isquemia distal y lograron la resolución completa y la perfusión normal de los dedos de los pies.
Por último, la rotura del CAU es una complicación poco frecuente; la extracción inmediata del fragmento roto puede salvar vidas. Los médicos deben estar atentos al riesgo de isquemia de las extremidades, incluso después de haber retirado el fragmento retenido. $\mathrm{Si}$, a pesar del tratamiento trombolítico y anticoagulante, persiste la isquemia distal, se puede recurrir a la aplicación tópica de nitroglicerina. El tratamiento trombolítico y anticoagulante puede causar hemorragia intracraneal.

\section{REFERENCIAS}

1. Butler-O'Hara M, Buzzard CJ, Reubens L, McDermott $\mathrm{MP}$, et al. A randomized trial comparing long-term and short-term use of umbilical venous catheters in premature infants with birth weights of less than 1251 grams. Pediatrics 2006;118(1):e25-35.

2. Cohen RS, Ramachandran P, Kim EH, Glasscock GF. Retrospective analysis of risks associated with an umbilical artery catheter system for continuous monitoring of arterial oxygen tension. J Perinatol 1995;15(3):195-8.

3. Hogan MJ. Neonatal vascular catheters and their complications. Radiol Clin North Am 1999;37(6):1109-25.

4. Choi SJ, Raziuddin D, Haller JO. Broken umbellical artery catheter: a report of two cases. Am J Dis Child 1977;131(5):595.

5. Vohra K, Shah P, Jhaveri R, Kanjilal D. Neonatal radiology casebook. Broken umbilical artery catheter: a rare occurrence. J Perinatol 1989;9(3):347-8.

6. Murphy KD, Le VA, Encarnacion CE, Palmaz JC. Transumbilical intravascular retrieval of an umbilical artery catheter. Pediatr Radiol 1995;25(Suppl 1):S178-9.

7. Schmidt B. The etiology, diagnosis and treatment of thrombotic disorders in newborn infants: a call for international and multi-institutional studies. Semin Perinatol 1997;21(1):86-9.

8. Hartmann J, Hussein A, Trowitzsch E, Becker J, et al. Treatment of neonatal thrombus formation with recombinant tissue plasminogen activator: six year's experience and review of the literature. Arch Dis Child Fetal Neonatal Ed 2001;85(1):F18-22.

9. Dursun A, Zenciroğlu A, Okumuş N. Neonatal arteriyel tromboz [Neonatal arterial thrombosis]. Çocuk Sağlığı ve Hastalıları Dergisi 2012;55:100-9. Turkish.

10. Mosalli R, Elbaz M, Paes B. Topical nitroglycerine for neonatal arterial associated peripheral ischemia following cannulation: a case report and comprehensive literature review. Case Rep Pediatr 2013;2013:608516. 